DOI 10.4171/JEMS/205

S. Fournais $\cdot$ B. Helffer

\title{
Bulk superconductivity in Type II superconductors near the second critical field
}

Received February 14, 2008 and in revised form July 31, 2008

Abstract. We consider superconductors of Type II near the transition from the 'bulk superconducting' to the 'surface superconducting' state. We prove a new $L^{\infty}$ estimate on the order parameter in the bulk, i.e. away from the boundary. This solves an open problem posed by Aftalion and Serfaty $[\mathrm{AS}]$.

\section{Introduction}

We consider a superconducting wire of cross section $\Omega \subset \mathbb{R}^{2}$, which we assume to be regular and bounded. The state of the material is described by the Ginzburg-Landau functional, which we write as

$\mathcal{G}(\psi, \mathbf{A})=\int_{\Omega}\left(\left|p_{\kappa \sigma \mathbf{A}} \psi\right|^{2}-\kappa^{2}|\psi|^{2}+\frac{\kappa^{2}}{2}|\psi|^{4}\right) d x+(\kappa \sigma)^{2} \int_{\Omega}|\operatorname{curl} \mathbf{A}-\beta|^{2} d x$.

We use the notation $p_{\mathbf{A}}=-i \nabla+\mathbf{A}$ for the magnetic gradient. In (1.1), $\kappa, \sigma$ are positive parameters, the wave function (order parameter) $\psi$ describes the superconducting properties of the given material and $(\kappa \sigma)$ curl $\mathbf{A}$ gives the induced magnetic field. The function $(\kappa \sigma) \beta$ represents the external magnetic field; in this paper we will for simplicity consider the case $\beta=1$, corresponding to a constant external field of intensity $\kappa \sigma$. We refer to deGe, Til for a general introduction to the physics of superconductivity and the Ginzburg-Landau model.

Consider the case $\sigma=\kappa / b$ with $b>0$. In the limit $\kappa \rightarrow \infty$ (called Type II limit), the following scenario presents itself. If $b<\Theta_{0}$, where $\Theta_{0} \approx 0.59$ is a universal constant, the only minimizer of $\mathcal{G}$ (for large $\kappa$ ) is the state $(\psi=0, \mathbf{A}=\mathbf{F}$ ), where curl $\mathbf{F}=1$ $[\mathrm{LuPa}, \mathrm{HePa}, \mathrm{FoHe} 1]$. This is interpreted as the loss of superconductivity for large external magnetic fields, and the value of $\sigma$ where this happens is denoted by $H_{C_{3}}$ and called the 'third critical field'.

S. Fournais: Department of Mathematical Sciences, University of Aarhus, Ny Munkegade, Building 1530, DK-8000 Aarhus C, Denmark; e-mail: fournais@imf.au.dk; on leave from: CNRS and Laboratoire de Mathématiques d'Orsay, Univ. Paris-Sud, Orsay Cedex, F-91405 France

B. Helffer: Laboratoire de Mathématiques UMR CNRS 8628, Université Paris-Sud, Bât. 425,

F-91405 Orsay Cedex, France; e-mail: bernard.helffer@math.u-psud.fr 
Physicists consider a second critical field, $H_{C_{2}}$, which can be described as follows (a precise definition being difficult to give). If $\sigma<H_{C_{2}}$, the material is in its superconducting state in (part of) the interior of the sample, whereas for $\sigma>H_{C_{2}}$ superconductivity is restricted to a narrow region near the boundary of $\Omega$. Various investigations show that-for large values of $\kappa$-this transition takes place near the value $\sigma=\kappa$, so even though this critical field is difficult to define, one expects that $H_{C_{2}} \approx \kappa[\mathrm{SS}, \mathrm{Pan}]$.

In this paper we will study what happens in the limit of large $\kappa$ - the Type II limitwhen $\sigma=\kappa / b$ with $b$ close to, but above the value 1 . In the terminology of superconductivity, this means that we study the parameter region close to but below the second critical field. In this region the so-called Abrikosov lattices of vortices are supposed to appear, but their description depends on a finer analysis than what will be carried out in the present paper (see [AS, $\mathrm{Alm}, \mathrm{SS}]$ for results in this direction).

Our main result, Theorem 2.1 below, gives for any $\delta>0$ the existence of a constant $C>0$ such that if $b>1, \kappa$ is large enough and $(\psi, \mathbf{A})$ is a minimizer of $\mathcal{G}$ then

$$
\|\psi\|_{L^{\infty}(\{x \in \Omega: \operatorname{dist}(x, \partial \Omega) \geq \delta\})} \leq C \sqrt{b-1} .
$$

This implies that in the interior of the sample, superconductivity is weak in the uniform norm as $b$ approaches 1 . Theorem 2.1 thereby answers a question posed in [SS p. 944 just below (1.20)] and more explicitly in [AS, List of open problems, p. 7].

Notice that $|\psi|$ is not expected to become small at the boundary when $b$ approaches the value 1 [Pan, AlHe].

We end this introduction by discussing the optimality of the estimate in (1.2). According to [SS, Theorem 1.4] (notice that the symbol $b$ in [SS] denotes a different quantity than in the present paper) there exists a continuous, decreasing function $g:[0,1] \rightarrow[0,1]$ such that if $b \geq 1$, if $\left(\psi_{\kappa}, \mathbf{A}_{\kappa}\right)_{\kappa \geq 1}$ denotes a family of minimizers of $\mathcal{G}$ with $\sigma=\kappa / b$ and if $\left\{B_{K}\right\}_{\kappa \geq 1}$ is a family of balls such that

(1) $\kappa \operatorname{radius}\left(B_{\kappa}\right) \rightarrow \infty$,

(2) $B_{\kappa} \cap \partial \Omega=0$,

then

$$
\frac{1}{\left|B_{\kappa}\right|} \int_{B_{\kappa}}\left|\psi_{\kappa}\right|^{4} d x \rightarrow g\left(b^{-1}\right) .
$$

Furthermore, the function $g$ satisfies the double bound

$$
\alpha\left(1-b^{-1}\right)^{2} \leq g\left(b^{-1}\right) \leq\left(1-b^{-1}\right)^{2}
$$

for some $\alpha$ with $0<\alpha<1$.

Combining (1.3) and (1.4) we see that $\left|\psi_{\kappa}\right|$ is of order $\sqrt{b-1}$ for $b$ near and above 1 and in the $L^{4}$-average sense given by 1.3 . More precisely, (by assuming the balls $B_{\kappa}$ to be contained in $\{x \in \Omega: \operatorname{dist}(x, \partial \Omega) \geq \delta\})$ we get the lower bound

$$
\liminf _{\kappa \rightarrow \infty}\left\|\psi_{\kappa}\right\|_{L^{\infty}(\{x \in \Omega: \operatorname{dist}(x, \partial \Omega) \geq \delta\})}^{4} \geq \liminf _{\kappa \rightarrow \infty}\left\|\psi_{\kappa}\right\|_{L^{\infty}\left(B_{\kappa}\right)}^{4} \geq g\left(b^{-1}\right) \geq \alpha \frac{(b-1)^{2}}{b^{2}},
$$

complementary to 1.2 , thereby yielding the optimality of the inequality. 


\section{Uniform estimates on the Ginzburg-Landau system}

We will study solutions to the Ginzburg-Landau equations, i.e. the stationary points of the GL-functional. For concreteness, let us assume that $\Omega \subset \mathbb{R}^{2}$ is a bounded, smooth and simply connected domain. These assumptions are likely to be unnecessarily restrictive, but they cover the most interesting cases and allow us to work without worrying about topological problems and regularity questions.

The Ginzburg-Landau equations are

$$
\begin{aligned}
& \left.\begin{array}{r}
p_{\kappa \sigma \mathbf{A}}^{2} \psi=\kappa^{2}\left(1-|\psi|^{2}\right) \psi \\
\operatorname{curl}^{2} \mathbf{A}=-\frac{1}{\kappa \sigma} \Re\left(\bar{\psi} p_{\kappa \sigma \mathbf{A}} \psi\right)
\end{array}\right\} \quad \text { in } \Omega ; \\
& \left.\begin{array}{rl}
v \cdot p_{\kappa \sigma \mathbf{A}} \psi & =0 \\
\operatorname{curl} \mathbf{A} & =1
\end{array}\right\} \quad \text { on } \partial \Omega .
\end{aligned}
$$

Here, $v$ is the interior unit normal vector to the boundary and for $\mathbf{A}=\left(A_{1}, A_{2}\right)$, curl $\mathbf{A}=$ $\partial_{x_{1}} A_{2}-\partial_{x_{2}} A_{1}$, and

$$
\operatorname{curl}^{2} \mathbf{A}=\left(\partial_{x_{2}}(\operatorname{curl} \mathbf{A}),-\partial_{x_{1}}(\operatorname{curl} \mathbf{A})\right) .
$$

Using gauge invariance it is no loss of generality to consider only (weak) solutions $(\psi, \mathbf{A})$ of (2.1) such that $(\psi, \mathbf{A}) \in H^{1}(\Omega, \mathbb{C}) \times H_{\text {div }}^{1}(\Omega)$, where

$$
H_{\operatorname{div}}^{1}(\Omega)=\left\{\mathcal{V}=\left(V_{1}, V_{2}\right) \in H^{1}(\Omega)^{2}: \operatorname{div} \mathcal{V}=0 \text { in } \Omega, \mathcal{V} \cdot \mathcal{v}=0 \text { on } \partial \Omega\right\}
$$

The space $H_{\text {div }}^{1}(\Omega)$ inherits the topology (norm) from $H^{1}\left(\Omega ; \mathbb{R}^{2}\right)$. We denote by $\mathbf{F}$ the unique vector potential in $H_{\text {div }}^{1}(\Omega)$ with $\operatorname{curl} \mathbf{F}=1$.

When we want to stress for which values of the parameters $\kappa, \sigma$ the system (2.1) is considered we will place these as indices. For instance, we will say that $(\psi, \mathbf{A})_{\kappa, \sigma}$ is a solution to 2.1.

Recall (see for instance [DGP] for a proof) that by the maximum principle we have the estimate

for all solutions to 2.1.

$$
\|\psi\|_{\infty} \leq 1
$$

We will write $|t|_{+}$for the 'positive part', i.e. the function

$$
\mathbb{R} \ni t \mapsto|t|_{+}:=\max (t, 0) .
$$

Our main result, the precise version of $(1.2)$, is as follows.

Theorem 2.1. There exists a constant $C_{\max }^{(2)}>0$ such that if $g_{1}: \mathbb{R}_{+} \rightarrow \mathbb{R}_{+}$with $g_{1}(\kappa) \rightarrow+\infty$ and $g_{1}(\kappa) / \kappa \rightarrow 0$ as $\kappa \rightarrow \infty$, and

$$
\omega_{\kappa}:=\left\{x \in \Omega: \operatorname{dist}(x, \partial \Omega) \geq g_{1}(\kappa) / \kappa\right\},
$$

then there exists a function $g_{2}: \mathbb{R}_{+} \rightarrow \mathbb{R}_{+}$with $g_{2}(\kappa) \rightarrow 0$ as $\kappa \rightarrow \infty$ such that

$$
\|\psi\|_{L^{\infty}\left(\omega_{\kappa}\right)} \leq C_{\max }^{(2)}|\kappa / \sigma-1|_{+}^{1 / 2}+g_{2}(\kappa)
$$

for all solutions $(\psi, \mathbf{A})_{\kappa, \sigma}$ to 2.1 with $\kappa \geq 1$. 
In the proof of Theorem 2.1 we will use the a priori estimates

$$
\|\mathbf{A}-\mathbf{F}\|_{W^{2, p}(\Omega)} \leq C_{p} \frac{1+\kappa \sigma+\kappa^{2}}{\kappa \sigma}\|\psi\|_{2}\|\psi\|_{\infty}
$$

valid for all $\kappa, \sigma>0$ and all solutions of (2.1), established in [FoHe2, (3.9)], and (see [FoHe2, (3.15)])

$$
\|\operatorname{curl} \mathbf{A}-1\|_{2} \leq \frac{C}{\sigma}\|\psi\|_{\infty}\|\psi\|_{2} .
$$

Proof of Theorem 2.1. By 2.3 the statement for $\kappa>2 \sigma$ is obvious. On the other hand, by Giorgi-Phillips [GiPh] (see also [FHBk] $)$, if

$$
\sigma \geq C_{G P} \max \{\kappa, 1\},
$$

then all solutions to 2.1 have $\psi=0$. Thus it suffices to consider the case

$$
C^{-1} \kappa \leq \sigma \leq C \kappa, \quad \kappa \geq 1
$$

Suppose for contradiction that 2.5) is false. Then, for all $N>0$ sufficiently large, there exists a sequence $\left\{\left(\psi_{n}, \mathbf{A}_{n}, \kappa_{n}, \sigma_{n}\right)\right\}_{n \in \mathbb{N}}$ with $\left(\psi_{n}, \mathbf{A}_{n}\right)_{\kappa_{n}, \sigma_{n}}$ a solution to 2.1 such that $\kappa_{n} \rightarrow \infty$ and

$$
\left\|\psi_{n}\right\|_{L^{\infty}\left(\omega_{\kappa_{n}}\right)} \geq N\left|\kappa_{n} / \sigma_{n}-1\right|_{+}^{1 / 2}+N^{-1} .
$$

Due to 2.9 we may assume, by possibly taking a subsequence, that

$$
\kappa_{n} / \sigma_{n} \rightarrow b \in\left[C^{-1}, C\right] .
$$

Using (2.6) and the compactness of the imbedding $W^{2, p}(\Omega) \rightarrow C^{1,1 / 2}(\bar{\Omega})$ for $p>2$ we may assume — by possibly taking a further subsequence — that

$$
\mathbf{A}_{n} \rightarrow \tilde{\mathbf{A}} \quad \text { in } C^{1,1 / 2}(\bar{\Omega}) .
$$

By 2.7) we have

$$
\operatorname{curl} \tilde{\mathbf{A}}=1 .
$$

Let $P_{n} \in \omega_{\kappa_{n}}$ be a point with $\left|\psi_{n}\left(P_{n}\right)\right|=\left\|\psi_{n}\right\|_{L^{\infty}\left(\omega_{\kappa_{n}}\right)}$. By 2.10) and 2.3 we therefore have

$$
N^{-1} \leq\left|\psi_{n}\left(P_{n}\right)\right| \leq 1
$$

After passing to a subsequence we assume that

$$
P_{n} \rightarrow P \in \bar{\Omega}
$$

We consider the scaled functions

$$
\begin{aligned}
\mathbf{a}_{n}(y) & :=\frac{\mathbf{A}_{n}\left(P_{n}+y / \sqrt{\kappa_{n} \sigma_{n}}\right)-\mathbf{A}_{n}\left(P_{n}\right)}{1 / \sqrt{\kappa_{n} \sigma_{n}}}, \\
\varphi_{n}(y) & :=e^{-i \sqrt{\kappa_{n} \sigma_{n}} \mathbf{A}_{n}\left(P_{n}\right) \cdot y} \psi_{n}\left(P_{n}+y / \sqrt{\kappa_{n} \sigma_{n}}\right) .
\end{aligned}
$$


Let $R>0$. Since $g_{1}(\kappa) \rightarrow+\infty, \mathbf{a}_{n}$ and $\varphi_{n}$ are defined on $B(0, R)$ for all $n$ sufficiently large. The equation for $\psi$ in 2.1a implies, since $\operatorname{div} \mathbf{a}_{n}=0$, that

$$
-\Delta \varphi_{n}-2 i \mathbf{a}_{n} \cdot \nabla \varphi_{n}+\left|\mathbf{a}_{n}\right|^{2} \varphi_{n}=\frac{\kappa_{n}}{\sigma_{n}}\left(1-\left|\varphi_{n}\right|^{2}\right) \varphi_{n}
$$

The convergences 2.12 and 2.15 imply that

$$
\mathbf{a}_{n}(y) \rightarrow \tilde{\mathbf{F}}(y):=D \tilde{\mathbf{A}}(P) y
$$

with convergence in $C^{1 / 2}(B(0, R))$ for all $R>0$. By 2.13) we find

$$
\operatorname{curl} \tilde{\mathbf{F}}=1
$$

The uniform (in $n$ ) boundedness of the coefficients of the equation (2.16) for $\varphi_{n}$ implies boundedness of $\left\{\varphi_{n}\right\} \subset W^{2, p}(B(0, R / 2))$ for all $p<\infty$ and all $R>1$. The compactness of the imbedding $W^{2, p} \rightarrow C^{1}$ (for $p>2$ ) implies that we can find a subsequence convergent in $C^{1}(B(0, R / 2))$. A diagonal sequence argument now gives the existence of a limiting function $\varphi \in L^{\infty}\left(\mathbb{R}^{2}\right)$ with

$$
N^{-1}+N|b-1|_{+}^{1 / 2} \leq\|\varphi\|_{L^{\infty}\left(\mathbb{R}^{2}\right)} \leq 1
$$

and

$$
(-i \nabla+\tilde{\mathbf{F}})^{2} \varphi=b\left(1-|\varphi|^{2}\right) \varphi .
$$

Since $\operatorname{curl} \tilde{\mathbf{F}}=1$, this contradicts Theorem 3.1 below if $N \geq C_{\max }$.

\section{Estimates for the global problem}

We will consider the following equation of Ginzburg-Landau type:

$$
p_{\mathbf{F}}^{2} u=b\left(1-|u|^{2}\right) u \quad \text { on } \mathbb{R}^{2},
$$

where $b \in \mathbb{R}$ is a parameter and $\mathbf{F}$ satisfies curl $\mathbf{F}=1$ in $\mathbb{R}^{2}$. For concreteness we use the gauge freedom of the problem to fix the choice

$$
\mathbf{F}=\left(-x_{2} / 2, x_{1} / 2\right)
$$

Theorem 3.1. (i) If $u \in L^{\infty}\left(\mathbb{R}^{2}\right)$ is a solution to $G L_{b}$ with $b \leq 1$, then $u=0$.

(ii) There exists a universal constant $C_{\max }>0$ such that if $u \in \bar{L}^{\infty}\left(\mathbb{R}^{2}\right)$ is a solution to $G L_{b}$ with $b>1$, then

$$
\|u\|_{\infty} \leq \min \left\{1, C_{\max } \sqrt{b-1}\right\}
$$


It is well-known [LuPa, FoHe2, $\mathrm{FHBk}]$ that the equation $\left(\overline{G L_{b}}\right)$ only admits trivial $L^{\infty}$ solution 11 if $b \leq 1$. Also, it is a standard consequence of the maximum principle that bounded solutions satisfy

$$
\|u\|_{\infty} \leq 1 .
$$

Thus only the second half of (3.1) needs to be proved.

Define

$$
S(b):=\left\{u \in L^{\infty}\left(\mathbb{R}^{2}\right): u \text { solves } G L_{b}\right\} .
$$

and

$$
M(b):=\sup _{u \in S(b)}\|u\|_{\infty} .
$$

The starting point is the following lemma.

Lemma 3.2. As $\epsilon \searrow 0$, we have the estimate

$$
M(1+\epsilon)=o(1) .
$$

Proof. The proof is by contraposition in the spirit of [FoHe2, LuPa]. Suppose that Lemma 3.2 is wrong. Then there exists a sequence $\left\{\epsilon_{n}\right\}_{n \in \mathbb{N}} \subset \mathbb{R}_{+}$with $\epsilon_{n} \rightarrow 0$ and an associated sequence $\phi_{n}$ of solutions to $\left(G L_{1+\epsilon_{n}}\right)$ with

$$
\left\|\phi_{h}\right\|_{\infty} \geq \delta>0 .
$$

Clearly, there will then exist a point $x_{n} \in \mathbb{R}^{2}$ with $\left|\phi_{n}\left(x_{n}\right)\right| \geq \delta / 2$. By magnetic translation invariance of $\left(G L_{b}\right)$ we may assume that $x_{n}=0$ for all $n$.

By elliptic regularity and 3.2$),\left\{\phi_{n}\right\}$ is bounded in $W^{2, p}(B(N))$ for all $N \in \mathbb{N}$ and all $p<\infty$. By compactness we can-for any given $s<2, p<\infty$ and $N \in \mathbb{N}$-find a convergent subsequence in $W^{s, p}(B(N))$.

By a diagonal sequence argument we get a $\phi \in W_{\text {loc }}^{s, p}\left(\mathbb{R}^{2}\right)$ and a subsequence, still denoted by $\left\{\phi_{n}\right\}$, such that

$$
\left\|\phi_{n}-\phi\right\|_{W^{s, p}(B(N))} \rightarrow 0
$$

for all $N$. In particular, we see that $\|\phi\|_{\infty} \leq 1$,

$$
|\phi(0)| \geq \delta / 2,
$$

and $\phi$ solves $\left(G L_{1}\right)$. But we know from [FoHe2, Proposition 4.1] (or part (i) of Theorem 3.1) that the only bounded solution to $\left(G L_{1}\right)$ is $\phi=0$, contradicting 3.7). This finishes the proof of Lemma 3.2 .

Proof of Theorem 3.1. Suppose for contradiction that there exists a sequence of solutions $\left\{\phi_{n}\right\}$ to $\left(G L_{1+\epsilon_{n}}\right)$ with

$$
\left\|\phi_{n}\right\|_{\infty} / \sqrt{\epsilon_{n}} \rightarrow \infty
$$

\footnotetext{
1 The case $b<1$ can be considered as a magnetic special case of a theorem by Shnol' [Sh, CFKS] (see also [Gl]).
} 
Define $\Lambda_{n}:=\left\|\phi_{n}\right\|_{\infty}$. By magnetic translation invariance, we may assume that $\left|\phi_{n}(0)\right| \geq$ $\Lambda_{n} / 2$. Consider the function $f_{n}:=\Lambda_{n}^{-1} \phi_{n}$. This function satisfies $\left\|f_{n}\right\|_{\infty} \leq 1$ and

$$
p_{\mathbf{F}}^{2} f_{n}=b_{n}\left(1-\Lambda_{n}^{2}\left|f_{n}\right|^{2}\right) f_{n}
$$

with $b_{n}:=1+\epsilon_{n}$. After possibly passing to a subsequence, we find

$$
f_{n} \rightarrow f \in W_{\mathrm{loc}}^{3 / 2,2}\left(\mathbb{R}^{2}\right) \hookrightarrow L_{\mathrm{loc}}^{\infty}\left(\mathbb{R}^{2}\right),
$$

where $f$ satisfies the lower bound

$$
1 / 2 \leq|f(0)| \leq\|f\|_{\infty} \leq 1 .
$$

Using Lemma 3.2 we get the limiting equation for $f$ :

$$
p_{\mathbf{F}}^{2} f=f .
$$

Thus $f$ lies in the lowest Landau band.

Let $\Pi_{0}$ be the projection on the lowest Landau band. This operator is given explicitly by the integral kernel (see for example [LSY, (2.10)])

$$
\Pi_{0}(x, y)=\frac{1}{2 \pi} e^{\frac{i}{2}\left(x_{1} y_{2}-x_{2} y_{1}\right)} e^{-\frac{1}{2}(x-y)^{2}} ;
$$

in particular, we see that $\Pi_{0}$ is a bounded operator on $L^{2}\left(\mathbb{R}^{2}\right)$ and on $L^{\infty}\left(\mathbb{R}^{2}\right)$. By interpolation, $\Pi_{0}$ is continuous on $L^{p}\left(\mathbb{R}^{2}\right)$ for all $p \in[2, \infty]$.

The boundedness of $f_{n}$ and elliptic regularity applied to 3.9 imply that the conditions of Proposition 3.3 below are satisfied. Therefore, an application of $\Pi_{0}$ to 3.9 ) shows that

$$
\left.0=\Pi_{0}\left\{\epsilon_{n} / \Lambda_{n}^{2}-\left|f_{n}\right|^{2}\right) f_{n}\right\}(x) \quad \text { for all } x \in \mathbb{R}^{2} .
$$

Using (3.8) and passing to the limit in 3.14 using (3.13) and dominated convergence, we obtain

$$
\Pi_{0}\left\{|f|^{2} f\right\}=0 .
$$

By Proposition 3.4 below we therefore conclude that $f=0$, which contradicts 3.11.

Proposition 3.3. Suppose that $f, p_{\mathbf{F}}^{2} f \in L^{\infty}\left(\mathbb{R}^{2}\right) \cap C\left(\mathbb{R}^{2}\right)$. Then

$$
\left(\Pi_{0}\left(p_{\mathbf{F}}^{2}-1\right) f\right)(x)=0 \quad \text { for all } x \in \mathbb{R}^{2} .
$$

Proof. By continuity of $f$, boundedness of $f$ and Gaussian decay of the kernel of $\Pi_{0}$ we find that $\Pi_{0} f$ is continuous. The same argument applies to $\Pi_{0}\left(p_{\mathbf{F}}^{2} f\right)$ and therefore $\Pi_{0}\left(p_{\mathbf{F}}^{2}-1\right) f$ is also continuous. Hence, it suffices to prove that

$$
\int \varphi(x)\left(\Pi_{0}\left(p_{\mathbf{F}}^{2}-1\right) f\right)(x) d x=0
$$

for all $\varphi \in C_{0}^{\infty}\left(\mathbb{R}^{2}\right)$, which is immediate. 
Proposition 3.4. Suppose that $f \in L^{\infty}\left(\mathbb{R}^{2}\right)$ satisfies

$$
p_{\mathbf{F}}^{2} f=f \quad \text { and } \quad \Pi_{0}\left(|f|^{2} f\right)=0 .
$$

Then $f=0$.

Below we will use the localization functions $\chi_{R}$ defined as follows. Let $\chi \in C^{\infty}(\mathbb{R})$ be even, non-increasing on $\mathbb{R}_{+}$and satisfy

$$
\chi(t)=1 \quad \text { for }|t| \leq 1, \quad \chi(t)=0 \quad \text { for }|t| \geq 3 / 2 .
$$

Define, for $R>0$ and $x \in \mathbb{R}^{2}$,

$$
\chi_{R}(x):=\chi(|x| / R) .
$$

Proof of Proposition 3.4 Since $f \in L^{\infty}\left(\mathbb{R}^{2}\right)$, we clearly have

$$
\int_{\{|x| \leq R\}}|f(x)|^{4} d x \leq C R^{2}
$$

for all $R>0$. We will prove that one can recursively improve the power of $R$ in 3.19, i.e. if the estimate

$$
\int_{\{|x| \leq R\}}|f(x)|^{4} d x \leq C R^{s}
$$

holds for all $R>1$ and some constant $C$, then there exists a new constant $C^{\prime}$ such that

$$
\int_{\{|x| \leq R\}}|f(x)|^{4} d x \leq C^{\prime} R^{s-1 / 2}
$$

for all $R>1$.

Since we get a negative power of $R$ after a finite number of steps, that will imply that $f=0$. Thus we only need to prove that 3.21 follows from 3.20 .

We calculate, using $\left(p_{\mathbf{F}}^{2}-1\right) f=0$,

$$
\begin{aligned}
\left\langle\left(p_{\mathbf{F}}^{2}-1\right) \chi_{R} f \mid \chi_{R} f\right\rangle & =\left\|\left(\nabla \chi_{R}\right) f\right\|_{2}^{2} \leq \frac{C}{R^{2}} \int_{\{|x| \leq 2 R\}}|f|^{2} d x \\
& \leq \frac{C^{\prime}}{R} \sqrt{\int_{\{|x| \leq 2 R\}}|f|^{4} d x} \leq C^{\prime \prime} R^{s / 2-1} .
\end{aligned}
$$

This gives, by $L^{2}$-projection, and dropping the primes on the constant,

$$
\left\|\Pi_{0}^{\perp}\left(\chi_{R} f\right)\right\|_{2}^{2} \leq C R^{s / 2-1},
$$

where we have introduced the notation $\Pi_{0}^{\perp}:=1-\Pi_{0}$. Since $\Pi_{0}$ is bounded from $L^{\infty}$ to $L^{\infty}$ we get $\left\|\Pi_{0}^{\perp}\left(\chi_{R} f\right)\right\|_{\infty} \leq C$, and by interpolation,

$$
\left\|\Pi_{0}^{\perp}\left(\chi_{R} f\right)\right\|_{4} \leq\left\|\Pi_{0}^{\perp}\left(\chi_{R} f\right)\right\|_{2}^{1 / 2}\left\|\Pi_{0}^{\perp}\left(\chi_{R} f\right)\right\|_{\infty}^{1 / 2} \leq C^{\prime} R^{s / 4-1 / 2} .
$$


We now write

$$
\begin{aligned}
\int \chi_{R}|f|^{4} d x & =\int \overline{\chi_{R} f} \Pi_{0}^{\perp}\left(|f|^{2} f\right) d x \\
& =\int \overline{\chi_{R} f} \Pi_{0}^{\perp} \chi_{2 R}\left(|f|^{2} f\right) d x+\int \overline{\chi_{R} f} \Pi_{0}^{\perp}\left(1-\chi_{2 R}\right)\left(|f|^{2} f\right) d x \\
& =\left\langle\Pi_{0}^{\perp}\left(\chi_{R} f\right) \mid \chi_{2 R}\left(|f|^{2} f\right)\right\rangle-\int \overline{\chi_{R} f} \Pi_{0}\left(1-\chi_{2 R}\right)\left(|f|^{2} f\right) d x
\end{aligned}
$$

Here we use the fact that $\Pi_{0}^{\perp}=1-\Pi_{0}$ and that $\chi_{R}\left(1-\chi_{2 R}\right)=0$ to get the last identity.

By Hölder's inequality combined with (3.24) and Lemma 3.5 below, we can therefore estimate

$$
\begin{aligned}
\int \chi_{R}|f|^{4} d x & \leq\left\|\Pi_{0}^{\perp}\left(\chi_{R} f\right)\right\|_{4}\left\|\chi_{2 R}|f|^{2} f\right\|_{4 / 3}+C e^{-R^{2} / 16} \\
& \leq C R^{s / 4-1 / 2} R^{3 s / 4}+C e^{-R^{2} / 16}
\end{aligned}
$$

Therefore, for some new constant $C>0$,

$$
\int_{\{|x| \leq R\}}|f|^{4} d x \leq C R^{s-1 / 2},
$$

which is 3.21). This finishes the proof.

Lemma 3.5. There exists a constant $C>0$ such that

$$
\int_{\mathbb{R}^{2}}\left\{\left(1-\chi_{2 R}\right) \Pi_{0} \chi_{R} u\right\} v d x \leq C e^{-R^{2} / 16}
$$

for all $u, v \in L^{\infty}\left(\mathbb{R}^{2}\right)$ with $\|u\|_{\infty},\|v\|_{\infty} \leq 1$ and all $R>1$.

Proof. Upon inserting the explicit integral kernel of $\Pi_{0}$, we get

$$
\begin{aligned}
\left|\int\left\{\left(1-\chi_{2 R}\right) \Pi_{0} \chi_{R} u\right\} v d x\right| & \leq C \int_{\{|x| \leq 3 R / 2\}} \int_{\{|y| \geq 2 R\}} e^{-|x-y|^{2} / 2} d x d y \\
& \leq C^{\prime} R^{2} \int_{\{|y| \geq 2 R\}} e^{-(|y|-3 R / 2)^{2} / 2} d y,
\end{aligned}
$$

from which the estimate is immediate.

Acknowledgments. Our interest in this problem was sparked by discussions with S. Serfaty. We would like to thank her for calling our attention to this interesting problem.

The two authors were partially supported by the ESF Scientific Programme in Spectral Theory and Partial Differential Equations (SPECT). SF is supported by a Skou Grant and a Young Elite Researcher Award from the Danish Research Council. 


\section{References}

[AS] Aftalion, A., Serfaty, S.: Lowest Landau level approach in superconductivity for the Abrikosov lattice close to $H_{C_{2}}$. Selecta Math. (N.S.) 13, 183-202 (2007) Zbl 1138.82034 MR 2361092

[Alm] Almog, Y.: Abrikosov lattices in finite domains. Comm. Math. Phys. 262, 677-702 (2006) Zbl 1106.35089 MR 2202308

[AlHe] Almog, Y., Helffer, B.: The distribution of surface superconductivity along the boundary: on a conjecture of X. B. Pan. SIAM J. Math. Anal. 38, 1715-1732 (2007) Zbl 1136.82042 MR 2299426

[CFKS] Cycon, H. L., Froese, R. G., Kirsch, W., Simon, B.: Schrödinger Operators with Application to Quantum Mechanics and Global Geometry. Texts and Monographs in Physics, Springer (1987) Zbl 0619.47005 MR 0883643

[deGe] de Gennes, P. G.: Superconductivity of Metals and Alloys. Benjamin (1966) Zbl 0138.22801

[DGP] Du, Q., Gunzburger, M. D., Peterson, J. S.: Analysis and approximation of the GinzburgLandau model of superconductivity. SIAM Rev. 34, 54-81 (1992) Zbl 0787.65091 MR 1156289

[FoHe1] Fournais, S., Helffer, B.: On the third critical field in Ginzburg-Landau theory. Comm. Math. Phys. 266, 153-196 (2006) Zbl 1107.58009 MR 2231969

[FoHe2] Fournais, S., Helffer, B.: Optimal uniform elliptic estimates for the Ginzburg-Landau system. In: Adventures in Mathematical Physics, Contemp. Math. 447, Amer. Math. Soc., 83-102 (2007) Zbl 1161.35042 MR 2423573

[FHBk] Fournais, S., Helffer, B.: Spectral Methods in Surface Superconductivity. Progr. Nonlinear Differential Equations Appl. 77, Birkhäuser (2010)

[GiPh] Giorgi, T., Phillips, D.: The breakdown of superconductivity due to strong fields for the Ginzburg-Landau model. SIAM J. Math. Anal. 30, 341-359 (1999) Zbl 0920.35058 MR 1926099

[Gl] Glazman, I. M.: On an application of the method of decomposition to multidimensional singular boundary problems. Mat. Sb. 35 (77), 231-246 (1954) (in Russian) Zbl 0056.34501 MR 0068089

[HePa] Helffer, B., Pan, X.-B.: Upper critical field and location of surface nucleation of superconductivity. Ann. Inst. H. Poincaré Anal. Non Linéaire 20, 145-181 (2003) Zbl 1060.35132 MR 1958165

[LSY] Lieb, E. H., Solovej, J. P., Yngvason, J.: Asymptotics of heavy atoms in high magnetic fields: II. Semiclassical regions. Comm. Math. Phys. 161, 77-124 (1994) Zbl 0807.47058 MR 1266071

[LuPa] Lu, K., Pan, X.-B.: Estimates of the upper critical field for the Ginzburg-Landau equations of superconductivity. Phys. D 127, 73-104 (1999) Zbl 0934.35174 MR 1678383

[Pan] Pan, X.-B.: Surface superconductivity in applied magnetic fields above $H_{C_{2}}$. Comm. Math. Phys. 228, 327-370 (2002) Zbl 1004.82020 MR 1911738

[SS] Sandier, E., Serfaty, S.: The decrease of bulk-superconductivity close to the second critical field in the Ginzburg-Landau model. SIAM J. Math. Anal. 34, 939-956 (2003) Zbl 1030.82015 MR 1969609

[Sh] Shnol', I. E.: On the behavior of the Schrödinger equation. Mat. Sb. 42, 273-286 (1957); erratum 46, 259 (1957) (in Russian) Zbl 0078.27904 MR 0125315

[Ti] Tinkham, M.: Introduction to Superconductivity. McGraw-Hill, New York (1975) 